\title{
Evening Lectures (E-1 - E-3)
}

\section{E-1 MAKI, Hisaji ${ }^{1}\left({ }^{1}\right.$ Dept. of Mol. Biol., Grad. School of Biol. Sci., Nara Inst. of Sci. and Tech.)}

\section{Origins of Spontaneous Mutations}

Spontaneous mutations and rearrangements of chromosomes are important cellular processes that lead to alteration of the genome structure and act as engines to drive the evolution of life. Analyses of spontaneous mutations occurring in various organisms, ranging from bacteriophages to human cells, have demonstrated that spontaneous mutations do not arise randomly in the genome sequence but possess a strong bias in their site distribution and in the types of alteration they cause within the DNA sequence. Each type of mutation shows a pattern of hotspot and cold-spot sites in a given sequence. The siteand type-specificities of spontaneous mutations imply that spontaneous mutagenesis, to a greater or lesseer degree, depends on the context of the DNA sequence. Probably, most of the events involved in spontaneous mutagenesis are affected by DNA topology, higher-order structures of DNA, and the extent of DNA transactions, such as transcription, replication, and recombination, on a given DNA sequence.

Spontaneous mutations are derived from various sources, including errors made during replication of undamaged template DNA, mutagenic nucleotide substrates, and endogenous DNA lesions. These sources differ in their frequencies and resultant mutations, and are differently affected by the DNA sequence, DNA transactions, and cellular metabolism. Organisms possess a variety of cellular functions to suppress spontaneous mutagenesis, and the specificity and effectiveness of each function strongly affect the pattern of spontaneous mutations. Base substitutions and single-base frameshifts, two major classes of spontaneous mutations, occur non-randomly throughout the genome. Within target DNA sequences there are hotspots for particular types of spontaneous mutations; outside of the hotspots, spontaneous mutations occur more randomly and much less frequently. Hotspot mutations are mostly due to endogenous DNA lesions rather than to replication errors. Recently, a novel class of mutagenic pathway that depends on short inverted repeats was found to be another important source of hotspot mutagenesis.

\section{E-2 OGASAWARA, Naotake ${ }^{1}\left({ }^{1} \mathrm{Grad}\right.$. Sch. of Information Sci., Nara Inst. of Sci. and Tech.)}

\section{Functional genomics of a model bacterium, Bacillus subtilis}

Complete genome sequences of 158 bacteria are now available in public data bases, and further genome sequencing is reported to be in progress for 179 bacterial species (http://www.ncbi.nlm.nih.gov/genomes/MICROBES/Complete.html). The genome sequences of several hundred microbes will be available in the very near future to explore microbial diversity.

The complete genome sequence of $B$. subtilis was determined by an international consortium of laboratories that had been engaging in B. subtilis biology. The $4,215 \mathrm{~kb}$ genome includes about 4,100 putative protein coding sequences. With acquisition of the complete genome sequence, it is a great challenge for biologists to transform the complete sequence information to information related to function. To this end, research consortia in Japan and Europe collected insertionally disrupted mutants of genes whose function had not been examined, and about 3,000 mutants covering essentially all the uncharacterized B. subtilis genes have been created. An important outcome of the project is the identification of novel essential genes that were not found in previous genetic studies. Combined with information from previous studies on essential nature of $B$. subtilis genes, it was concluded that 271 genes are indispensable for the $B$. subtilis growth in $\mathrm{LB}$ medium at $37^{\circ} \mathrm{C}$ (Kobayashi et al., Proc Natl Acad Sci U S A 100, 4678-4683, 2003).

The genome sequence indicated that in addition to the major sigma factor (sigA), the $B$. subtilis genome has 16 secondary sigma factors, which are dispensable for growth, but activate groups of genes under specific cellular conditions. In addition, about 250 DNA binding transcriptional regulators are further involved in the regulatory network of gene expression in B. subtilis. As a complementary approach to reverse genetics of individual genes, expression profiles of knockout mutants of regulator genes are being analyzed in the world, to develop a comprehensive picture of the regulatory network. Among transcriptional regulators, the two-component signal transduction system is an important bacterial mechanism for sensing and responding to various environmental stimuli, thus elucidation of target genes of the $B$. subtilis 35 two-component regulators has been one of the focuses of the genome function analysis. With combination of various approaches including comparative genomics, we now know or able to predict the targets of most of the regulators. Furthermore, interesting links between two-component and ECF sigma signal transduction systems have been revealed.

\section{E-3 KOHARA, Yuji ${ }^{1}\left({ }^{1}\right.$ Natl. Inst. Genet.)}

\section{Functional genomics of the nematode C.elegans}

The nematode C.elegans is one of the simplest multi-cellular organisms. It is an elegant organism, which was chosen for the research of development, neurobiology and behavior by Sydney Brenner in early 60's but was once a joke organism. Since then, fundamental studies on this tiny organism have been made including the entire cell lineage from a fertilized egg to adult, the complete diagram of neural network by mid 80's and the entire genome sequence in late 90 's, leading to make C.elegans a superstar of the model organisms. Looking at development of C.elegans through microscope, many people dream of the understanding of the entire process from genome to an papism, the computer understanding of the entire process from genome to an organism, the computer the genome analysis in C.elegans. Here, I will summarize the current situation of the genome analysis in C.elegans.

functional genomics of C.elegans.
1) The genome structure: C.elegans genome is $100 \mathrm{Mb}$ in size, one of the smallest genomes of multi-cellular organisms, on which some 19,000 genes have been predicted. We have identified the correct gene structure of about 12,000 genes by aligning EST sequences along the genome sequence, together with the alternative splicing patterns (av. $1.7 \mathrm{mRNA}$ and 1.4 protein from a gene).

2) Expression and function: We have performed whole mount in situ hybridization systematically to examine the distribution of mRNAs of these genes. Clustering of the patterns produced sets of genes that might be regulated in the same mechanisms. Analysis of the 5'-upstream sequences of these genes is in progress to abstract the candidates for regulatory sequences. We think that function analysis based on the expression pattern is powerful and we are applying the strategy to early embryogenesis that is governed mostly by maternally supplied gene products. Many of the important genes in the process show the expression pattern such that mRNA is present in oocytes and disappears quickly or localizes after fertilization. Such a class of genes consists of $8 \%$ of all genes (1000 cDNAs), to which we have performed RNAi experiments and 4D microscopic analysis to elucidate the stage of their function and ultimately reconstruct the network of gene regulation in very early embryogenesis. To this end, we are also raising gene regulation in very early embryogenesis. To
antibodies to these gene products systematically.

antibodies to these gene products systematically.
3) Comparative genomics: Comparison between the genomes of closely related species is powerful to examine the candidate motifs and regulatory sequences. So far, the genomes of 2 other nematode has been sequenced, whose shape and development are very similar with C.elegans. We are going to sequence another nematode whose developmental pattern is partly different from C.elegans in order to compare the gene system governing the process.

It was perhaps Sydney Brenner who said "The word, functional genomics, is popular these days but we knew another, simpler and more appropriate one long before. It's genetics." No doubt that the concept of genetics is still essential in the post-sequence era. 